\title{
Hamster Ductus Deferens Leiomyosarcoma
}

National Cancer Institute

\section{Source}

National Cancer Institute. Hamster Ductus Deferens Leiomyosarcoma. NCI Thesaurus.

Code C134567.

Ductus deferens leiomyosarcoma that occurs in a hamster. 